\title{
Multi-modality Management of an Intradural- Extramedullary Hemangioblastoma: A Case Report
}

\author{
Benjamin M. Zussman, BS', David L. Penn, MS', Shiveindra Jeyamohan, MD², \\ Maria Werner-Wasik, MD³ ${ }^{3}$ David W. Andrews, MD , James S. Harrop, MD ${ }^{4}$ \\ 'Jefferson Medical College, Thomas Jefferson University, Philadelphia, PA \\ ${ }^{2}$ Department of Neurosurgery, Albany Medical College, Albany, NY \\ ${ }^{3}$ Department of Radiation Oncology, Thomas Jefferson University, Philadelphia, PA \\ ${ }^{4}$ Department of Neurological Surgery, Thomas Jefferson University, Philadelphia, PA
}

\section{Abstract}

Objective

Stereotactic spinal radiosurgery may offer a complementary adjunct to microsurgery for patients with limited surgical candidacy due to significant medical comorbidities, aggressive or recurring tumors, or surgically inaccessible lesions. Our objective was to illustrate Intensity-Modulated Radiation Therapy (IMRT) treatment schemes in conjunction with surgical therapy for aggressive spinal neoplasms.

\section{Methods}

\section{Case Report}

A 79-year-old man with severe progressive tetraparesis had an intradural-extramedullary mass at the C4-C5 level with severe spinal cord compression that extended out the foramen and anterior to the vertebral artery. Subtotal microsurgical resection was performed, completely decompressing the spinal cord and sparing the structures exiting the left neural foramen. Imaging at five-months follow-up showed tumor progression. Stereotactic radiosurgery was performed which arrested tumor growth.

\section{Results}

Multimodality treatment resulted in significant neurological recovery without repeat invasive techniques.

\section{Conclusion}

This case demonstrates that microsurgery followed by stereotactic spinal radiosurgery is an effective approach for the treatment of intradural-extramedullary hemangioblastoma. Multiple treatment modalities provide for individualized patient care.

\section{Key Words}

Hemangioblastoma; Radiosurgery; Spine; Intradural; Extramedullary

\section{Abbreviations \\ Computed Tomography = CT; Intensity-Modulated Radiation Therapy $=$ IMRT}

\section{Introduction}

Hemangioblastomas are highly vascular, histologically benign tumors of the neuroaxis, which may develop idiopathically or in association with von Hippel-Lindau disease. ${ }^{1}$ Less than $20 \%$ of hemangioblastomas are located in the spinal cord; ${ }^{1,8}$ these lesions are typically intradural,

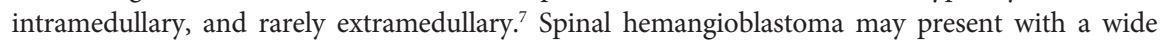
variety of clinical symptoms, ranging from mild neurological symptoms to paralysis. ${ }^{1}$

A 79-year-old gentleman with a four-month history of progressively deteriorating tetraparesis was transferred to our institution. The patient's previous medical history was significant for cardiac arrhythmia, Type-1 diabetes mellitus, hypertension, hyperlipidemia, and a pacemaker, but not von Hippel-Lindau's disease.

Upon admission, neurological examination demonstrated upper extremity strength of $0-1 / 5$ bilaterally and lower extremity strength of $2 / 5$ on the right and $0 / 5$ on the left. The patient had decreased sensation to light touch and pain below spinal level C4, 2/5 reflexes in all extremities, down-going toes, bilateral Hoffman's, and no clonus. A cervical myelogram showed a complete intradural block at C4-C5. Computed Tomography (CT) of the cervical spine identified an intradural-extramedullary mass at C4-C5 with severe spinal cord compression (Figures 1 and 2).

A posterior laminectomy and resection of the intradural tumor was performed. The spinal cord was well decompressed but the tumor extended out of the foramen and circumferential to the vertebral artery. Since gross total resection would have entailed sacrificing the nerve root and vertebral artery in this elderly patient, a subtotal resection was performed with accomplishment of the goal of decompressing the spinal cord.

Postoperatively the patient demonstrated significant neurological improvement in his upper and lower extremities; he returned to ambulating without assistive devices and independent living. Serial imaging at five-month CT studies, however, revealed progressive growth of the tumor in the left neural foramen towards the canal (Figure 3), but no spinal cord compression. A repeat-surgery would require significant osseous resection and dissection off of the vertebral artery. Due to the patient's comorbidities and potential surgical morbidities we opted for an Intensity-Modulated Radiation Therapy (IMRT) approach.

Stereotactic IMRT was delivered in a single fraction (lesion volume $=10.39 \mathrm{cc}$ and dose $=$ 12 Gy) (Figure 4). The percentage of the lesion volume enclosed by the prescribed iso-surface was $97 \%$. The patient tolerated the procedure without difficulty. At two-years post-radiation he demonstrated strength of $4-4+/ 5$ in each extremity and his incisions were completely healed, with no further progression of the tumor.

\section{Discussion}

While open surgery is the commonest treatment for spinal neoplasms, spinal hemangioblastomas may be well treated by IMRT if there is ample room between the spinal cord and radiation target. Ryu et $\mathrm{al}^{5}$ studied the effect of stereotactic radiosurgery on 10 intramedullary spinal tumors. They reported no significant treatment-related 


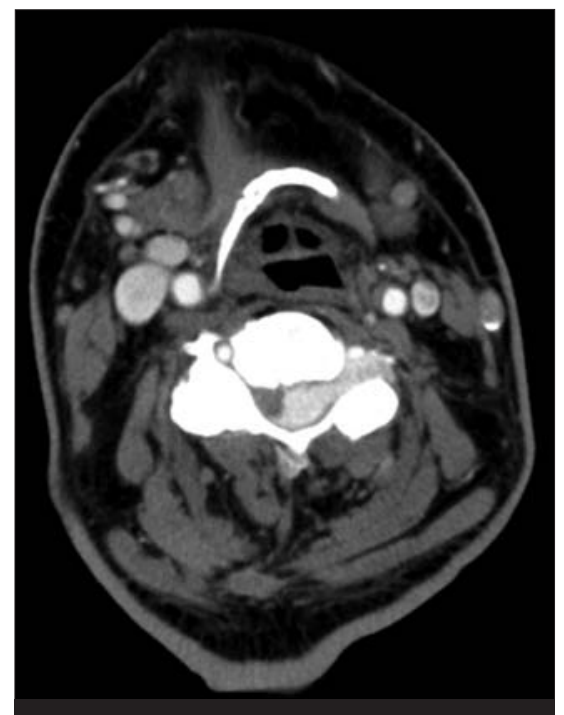

Figure 1

Preoperative axial CT image with contrast at the C4-C5 spinal level. Widening of the left neural foramen secondary to the soft tissue mass is apparent.

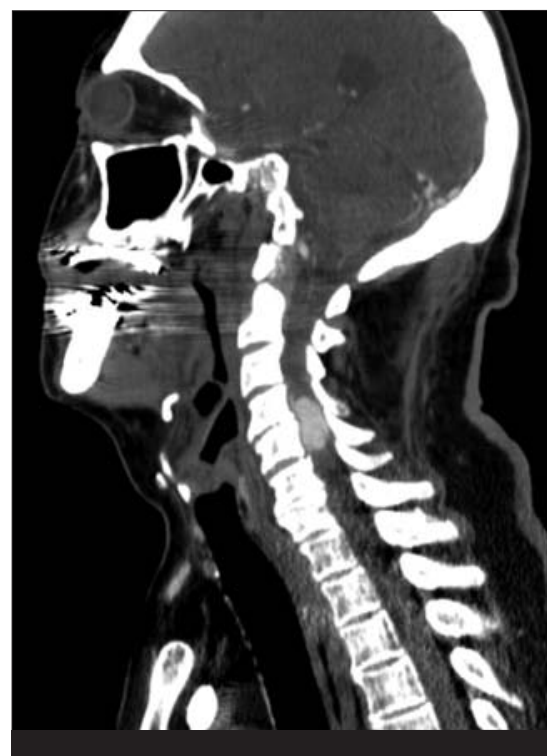

Figure 2

Preoperative saggital CT image.

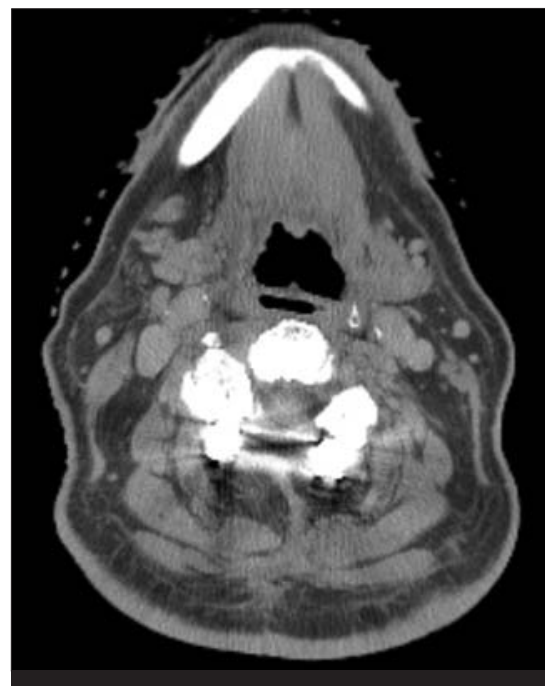

Figure 3

Postoperative axial CT image with contrast at the C4-C5 spinal level. An enhancing mass is visualized within the left $\mathrm{C} 4$-C5 neural foramen and lateral spinal canal. There is no spinal stenosis or cord compression.
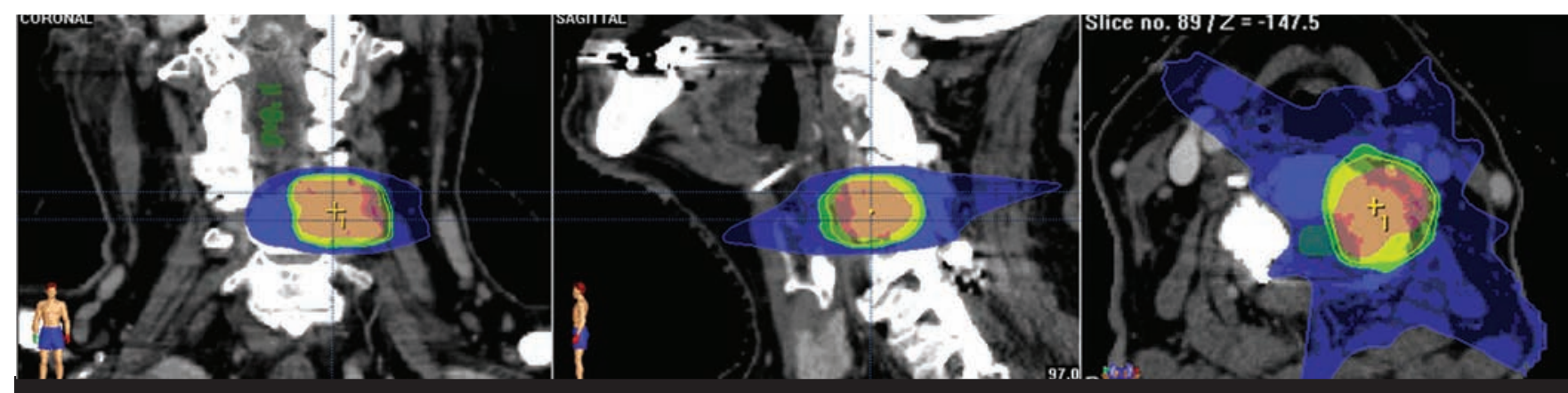

Figure 4

Intensity-Modulated Radiation Therapy (IMRT) treatment planning screen shot. Radiation delivery conforms to the 3-dimensional shape of the tumor by modulating the intensity of the radiation beam to focus the highest dose on the tumor while minimizing radiation exposure to the surrounding normal structures. Note the excellent margin of approximately $2 \mathrm{~mm}$ between the spinal cord and hemangioblastoma.

complications and at a mean of one-year follow-up all tumors were either stable or smaller. Although these tumors often progress slowly and changes might not be demonstrated at one-year follow-up, they concluded that stereotactic radiosurgery for intramedullary spinal tumors is feasible and safe. Moss et $\mathrm{al}^{4}$ studied 16 spinal hemangioblastomas treated with stereotactic radiosurgery and found that 15 of them either decreased in size or remained stable. These studies demonstrate that treatment of spinal neoplasms and hemangioblastomas with high-dose radiation yields adequate local tumor control.

IMRT is also a potential alternative for patients with contraindications to surgery, such as age, medical comorbidities, or lesion anatomy or location. Dodd et $\mathrm{al}^{2}$ studied the effect of image-guided radiosurgical ablation on benign spinal neoplasms in 55 patients with contraindications to microsurgical resection and found that tumors in 52 patients were either stable or smaller at a mean follow-up of three years. They concluded that radiosurgery is a useful adjunct to the neurosurgical "tool kit" for managing spinal neoplasms in patients with contraindications to open surgery.

In this case microsurgery was initially chosen primarily to immediately decompress the spinal cord. IMRT was contraindicated because there was not an adequate margin between the spinal cord and the hemangioblastoma to avoid risking radiation-induced spinal cord necrosis 
or myelopathy. Although image-guided stereotactic radiosurgery has only recently been applied to spinal hemangioblastomas, a few cases of severe radiation myelopathy secondary to IMRT have been reported, ${ }^{2}$ and the precise radiation thresholds and treatment schemes that cause the spinal cord to develop radiation myelopathy have not been elucidated. ${ }^{3}$ It is the author's preference to use IMRT for extramedullary-intradural spinal neoplasms only when there is ample margin between the spinal cord and tumor to avoid this complication. In this case microsurgery created an excellent margin between the spinal cord and remaining hemangioblastoma such that should tumor progression occur, IMRT would be a viable option. Tumor progression did occur and, in consideration of the patient's significant medical comorbidities, IMRT was chosen and ultimately arrested tumor progression.

This case highlights the advantages of a multi-modality scheme of IMRT in conjunction with microsurgical therapy for the management of aggressive spinal neoplasms, and specifically intradural-extramedullary hemangioblastomas. Technological advances have allowed physicians to increasingly individualize and optimize patient care, and in this case the combination of different therapies including invasive and non-invasive procedures to create a multi-modality treatment regimen allowed our team to better consider the patient's anatomy and medical comorbidities without compromising clinical results. ${ }^{6}$

\section{References}

1. Boughey AM, Fletcher NA, Harding AE. Central nervous system haemangioblastoma: a clinical and genetic study of 52 cases. J Neurol Neurosurg Psychiatry. 1990;53(8):644-648.

2. Dodd RL, Ryu MR, Kamnerdsupaphon P, Gibbs IC, Chang SD,Jr, Adler JR,Jr. CyberKnife radiosurgery for benign intradural extramedullary spinal tumors. Neurosurgery. 2006;58(4):674-85; discussion 674-85. 10.1227/01. NEU.0000204128.84742.8F.

3. Dropcho EJ. Neurotoxicity of radiation therapy. Neurol Clin 2010;28(1):217-234. 10.1016/j.ncl.2009.09.008.

4. Moss JM, Choi CY, Adler JR,Jr, Soltys SG, Gibbs IC, Chang SD. Stereotactic radiosurgical treatment of cranial and spinal hemangioblastomas. Neurosurgery. 2009;65(1):79-85; discussion 85. 10.1227/01.NEU.0000348015.51685.D2.

5. Ryu SI, Kim DH, Chang SD. Stereotactic radiosurgery for hemangiomas and ependymomas of the spinal cord. Neurosurg Focus. 2003;15(5):E10.

6. Saraceni C, Harrop JS. Spinal meningioma: chronicles of contemporary neurosurgical diagnosis and management. Clin Neurol Neurosurg. 2009;111(3):221-226. 10.1016/j. clineuro.2008.10.018

7. Taniguchi S, Ogikubo $\mathrm{O}$, Nakamura $\mathrm{T}$, et al. A rare case of extramedullary-intradural hemangioblastoma in the thoracic spine. Spine (Phila Pa 1976). 2009;34(26):E969-72. 10.1097/ BRS.0b013e3181b8e4f4.

8. Zimmermann M, Seifert V, Schreyer F, Stolke D, Dietz H. Hemangioblastoma: description of a disease picture and report of 41 cases. Zentralbl Neurochir. 1997;58(1):1-6.

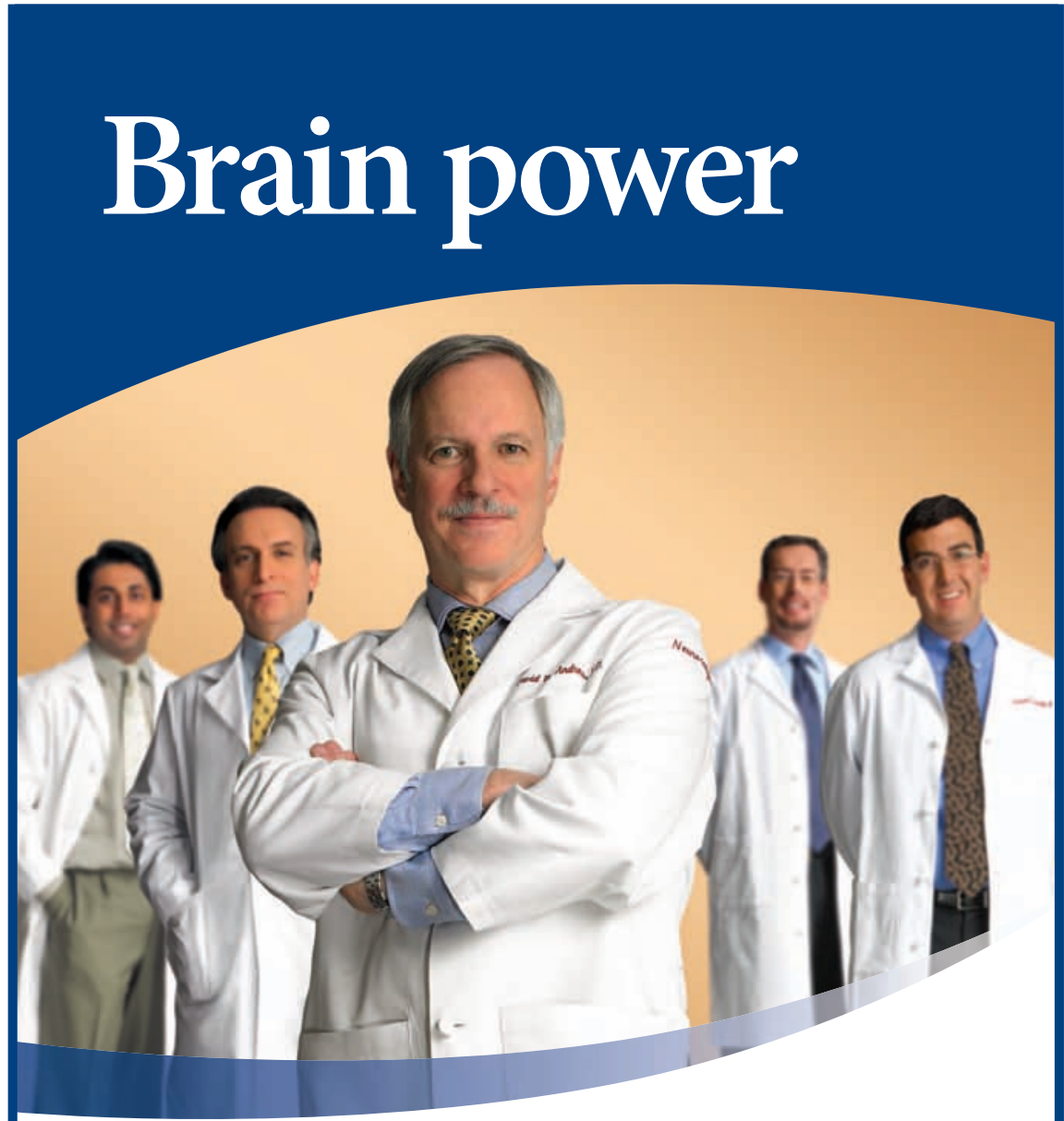

Jefferson neurosurgeons have helped to write the textbooks and set training standards referenced by other doctors around the world. No wonder our surgeons treat the largest combined volume of tumors, aneurysms and AVMs in the Philadelphia region.

The region's only hospital with an experienced, specialized staff dedicated to neuroscience.

Offices in Center City Philadelphia, Langhorne and Voorhees.

Most insurance plans accepted, including Medicare.

\section{Jefferson.}

Hospital for Neuroscience

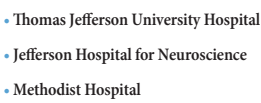

